Pak. j. sci. ind. res. Ser. A: phys. sci. 201659 (1) 30-33

\title{
Remote Controlling and Monitoring of Microscopic Slides
}

\author{
Ghulam Mustafa $^{\mathrm{a} *}$, Muhammad Tahir Qadri ${ }^{\mathrm{b}}$ and Umar Daraz \\ ${ }^{a}$ PCSIR Laboratories Complex, Karachi-75280, Pakistan \\ ${ }^{b}$ Department of Electronics Engineering, Sir Syed University of Engineering \& Technology, Karachi, Pakistan \\ 'Pathological Department of Civil Hospital, Karachi, Pakistan
}

(received December 18, 2014; revised May 11, 2015; accepted May 21, 2015)

\begin{abstract}
Remotely controlled microscopic slide was designed using especial Graphical User Interface (GUI) which interfaces the user at remote location with the real microscope using site and the user can easily view and control the slide present on the microscope's stage. Precise motors have been used to allow the movement in all the three dimensions required by a pathologist. The pathologist can easily access these slides from any remote location and so the physical presence of the pathologist is now made easy. This invention would increase the health care efficiency by reducing the time and cost of diagnosis, making it very easy to get the expert's opinion and supporting the pathologist to relocate himself for his work. The microscope is controlled with computer with an attractive Graphical User Interface (GUI), through which a pathologist can easily monitor, control and record the image of a slide. The pathologist can now do his work regardless of his location, time, cost and physically presence of lab equipment. The technology will help the specialist in viewing the patients slide from any location in the world. He would be able to monitor and control the stage. This will also help the pathological laboratories in getting opinion from senior pathologist who are present at any far location in the world. This system also reduces the life risks of the patients.
\end{abstract}

Keywords: pathology, remote monitoring, microscope, motor control, LabVIEW, Arduino, teamviewer

\section{Introduction}

Health care is the most important sector these days and many scientists are busy in their research using the new era technologies to provide health facilities to human beings (John et al., 2009; Kamel- Boullos and Wheeler 2007; Eysenbach, 2001). In the recent time there is a rapid development in information technologies i.e., growth of internet through high speed networks, communication technologies, web technologies, etc. Combination of new age technologies gave birth of a new field which is remote monitoring and controlling which rises the technological change in the field of control industries, communication industries, automotive industries, medical, agriculture etc. The aim of present study is to facilitate the personnels working in medical field, specifically a pathologist and to reduce the time of diagnosis of a disease. This research paper introduces a new solution for monitoring and controlling the microscopic slides regardless of the location of the pathologist.

Facilitating a pathologist has always remained an important issue of discussion, because in an emergency diagnosis can be delayed due to unavailability of the pathologist which is a high risk of patient's life. So there have been several methods used previously to resolve this matter.

*Author for correspondence; E-mail: mustafabme@gmail.com
But monitoring and controlling the slides from any location in the world is a great achievement. This research shows the real implementation of the method that could be used for remote monitoring of slides and control of the stage in up-down, right-left and in and out movements.

The machine is composed of a visualization system which is interfaced with the microscope, a control board that controls and interfaces the user interface with motors, a graphical user interface which contains the graphical push buttons providing the control in three dimensions and a software through which the doctor can interact with the user interface remotely.

This is a low cost and very effective method by which the time to diagnose a disease would be readily decreased. Microscopic examination cost is very high for the underdeveloped countries where the slides are being sent to other cities to the specialist. Now, using this technique the pathologist can give his report in a few minutes. Also this will reduce the health care cost that was previously bared by the patients and the pathological laboratories. Also the pathologists will be free from moving into different labs and can easily perform their task from any location even from their home. This will facilitate them physically as well as economically. 


\section{Materials and Methods}

This system model is described as follows: The system consists of five parts. The first part is the image acquisition whose purpose is to acquire the real time video signal using LabVIEW (NILS, 2014) based graphical user interface (GUI). The second part is to process the video signal using image processing tools in LabVIEW. The GUI is designed for this purpose which is the medium of communication between the physician and the microscopic unit. The third part is the design of controlling circuitry in order to control the movement of microscopic slides using stepper motors. The fourth part is the arduino (Arduino, 2014a) based controlling unit which is interfaced with the GUI to move the slides accordingly. The last part of the system is to publish the GUI on internet for remote monitoring and controlling of the microscopic slides. The overall block diagram of the system is illustrated in Fig. 1.

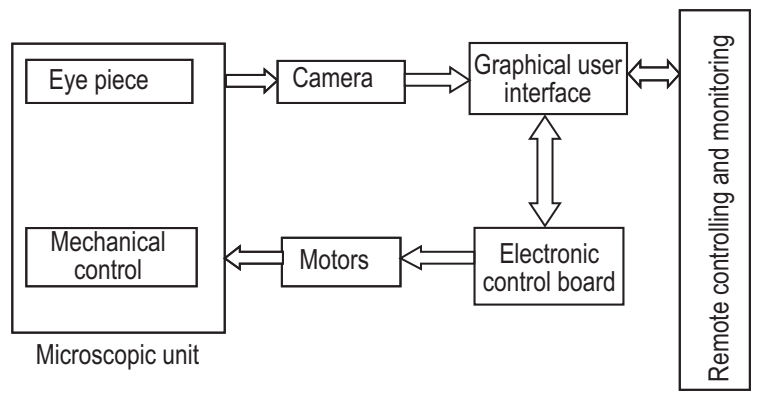

Fig. 1. Overall block diagram of the system.

Image acquisition. The most important part of the microscope is the eye piece from where the image of the slide is seen by the pathologist. In order to acquire the image from the eye piece, a digital camera of high resolution is attached on the eye piece. The camera is interfaced at the USB port of the system and is able to transmit live video of the slide to the attached computer. For this purpose LabVIEW software (NILS, 2014) is used.

To interface the camera in LabVIEW an additional tool kit is required. LabVIEW provides a solution known as vision acquisition software (NIVAS, 2014) which makes it very easy to interface any USB camera in LabVIEW. After interfacing the camera the live video of the slide of microscope was viewed as shown in Fig. 2.

LabVIEW based GUI. To get the user's input and translate it into machine understandable format, an interface is required to communicate. This interface must be easy to understand and simple to use, so that the user can perform its task very easily. The interface developed here is named

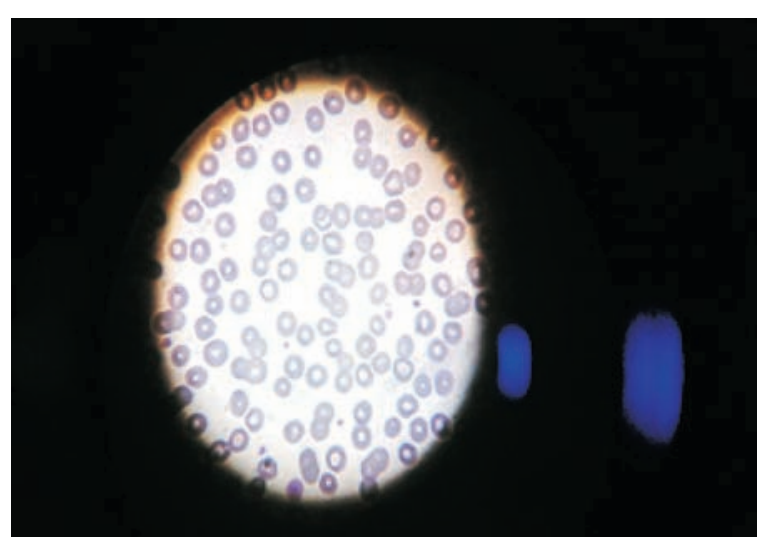

Fig. 2. Snapshot of the microscopic slide video.

as a graphical user interface. This is the main link of the whole system as it provides the communication between the user's interface and the central control board, which is arduinio based system. The task of the GUI is to handle the following operations.

i. Image acquisition.

ii. To process the user's input.

iii. Communicating the data to the control board.

The image was acquired in LabVIEW using the Vision Acquisition Software, which provides the opportunity to interface any USB imaging device having direct show support. In order to view the slide continuously, the camera is used in a live video display mode.

As the slide can be moved in all the three dimensions, therefore, the user is provided with the control buttons on the front panel to move it accordingly. These controls are simply made by using push buttons. The GUI contains the push buttons to adjust the position of slide and a control which displays the live video of the slide. The hardware interfaced gets the input corresponding specific button pressed by the user, as illustrated in Fig. 3 .

Finally, the control board is made by using Arduino mega2560 (Arduino, 2014b) microcontroller board. This board is interfaced with LabVIEW on HyperTerminal (HTS, 2014) on USB port.

Microscopic slides controlling. The slide is placed on the microscope called as 'Stage'. After placing a slide on the stage of microscope, the stage is moved 'up', 'down', 'in' and 'out' and the slide can be moved in right and left directions. These all actions are performed using the mechanical knobs present for the tasks in microscope. In order to control these movements through a computer, especial stepper motors are required to be attached with 


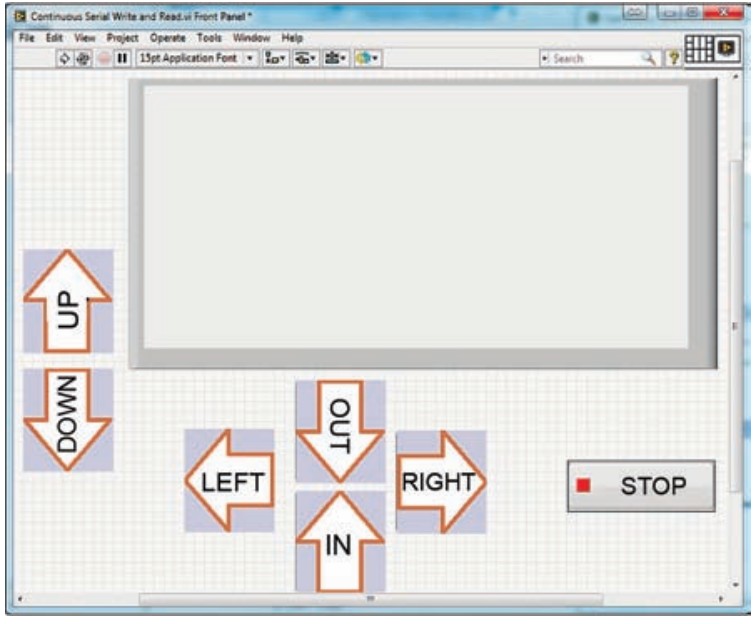

Fig. 3. LabVIEW based GUI.

individual knob. Since our requirement is two dimension accurate movement in each dimension. A stepper motor of $1.8^{\circ}$ step is feasible for the task. The torque of the motor is dependent upon the mechanical force required to move the knob. An easily available stepper motor can provide enough torque to rotate it.

Electronic circuit is required to control the rotation of the motors. 'Arduino Motor Control Shield' was used to control the stepper's movement. The Arduino shield can be easily attached with the Arduino main board and provide the additional library of motor control through which the user can control the acceleration, direction and torque of the motor easily.

The arduino shield has the capacity to control two stepper motors at a time. Therefore, an additional circuit developed on ULN2003 was used which was directly attached with the Arduino main board and the stepper motor. With these attachments it became easy to have a control on the movement of slide of microscope. In order to view the sample of the slide especial arrangement has to be made through which the slide moves accurately and precisely.

In order to control the slide's direction on the stage, an especial control circuitry is required to accurately move the slide in three dimensions. The slide placed by the technician on the stage of microscope could only be seen easily by the doctor when he can view the whole span of the slide. In order to achieve the target, three stepper motors were used to control the up-down, right-left and in-out motion of it. Each stepper motor with the accuracy of $1.8^{\circ}$ was used, which was providing accurate linear motion in all dimensions.
Arduino based controlling unit. A central control board was required to get the signal from the user and then to control the required motor in specified direction and with specific speed. There are several options to perform the task, but an Arduino board is a good option. The Arduino board is developed around Atmega AVR Microcontrollers and provides the opportunity to use its ports. The Arduino also provides an easy to learn and programme code that could be used to run the microcontroller used in the board. The Arduino board is USB powered and is supplied with its own compiler, so it gets very easy for the user to write, compile and upload the code. The Arduino Board comes in variety and the user can select specific board according to their requirements. Due to Arduino Motor Shield with a facility to drive three stepper at a time Arduino mega 2560 board was used in the present study. This board has 8 bit microcontroller having 86 digital I/O pins to cope with present requirement.

The Arduino mega board was programmed to control the motor and to communicate with the GUI. The Central Control board communicates with computer and gets the control signals from the user and transmits this into the motor control signal to specific motor.

Remote monitoring and controlling of the slides. Accurate software through which the remote monitoring and controlling could be done was the most important part of this solution. To access any system remotely it is always important to provide a secure access so that only that person can use it having the full authority. Remote computer controlling software was used for this purpose which provides a secure access to the user present at the remote location. There are many softwares available in the market that provides the remote desktop sharing option, they also provide the control of the desktop. Team viewer software (TVS) is one of them. Team viewer software was used to share the desktop of the computer on which the GUI was present. Figure 4 shows the front panel view from the remote location.

\section{Results and Discussion}

The operation of the complete remote controlled microscope is divided into two ends. One end is the microscope interfaced with the $\mathrm{PC}$ and the other end is the remote computer on which the pathologist is present to view the slide.

At the first end a PC is interfaced with the microscope through its camera and Arduino board connected at the 


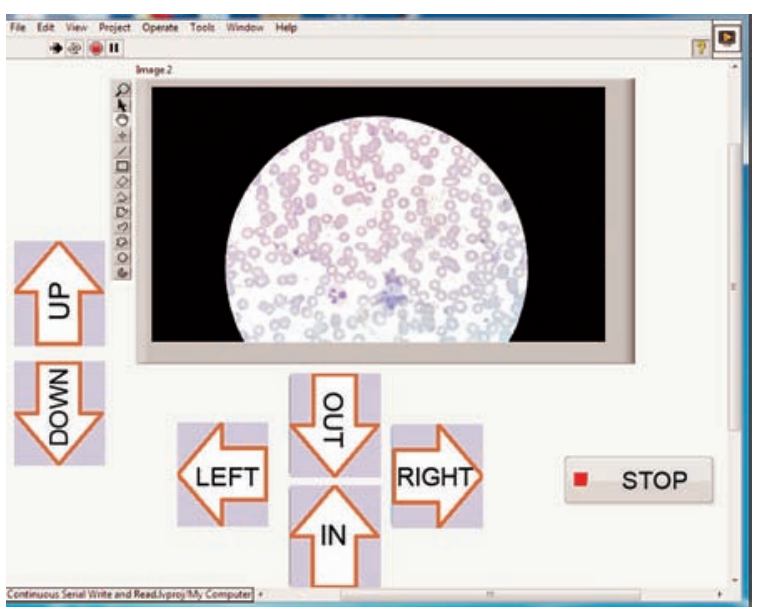

Fig. 4. Front panel to view and control the microscopic slides from remote location.

USB port of the PC, the graphical user interfaces the hardware which displays the live video of the slide to be examined. A remote desktop sharing and control software must be installed on both sides so that the screen could be easily shared, also a fast internet connection is required to connect this system with the remote system and to give a look of live monitoring of the slide.

The other end from which the pathologist views the slide does not need anything other than a remote controlling software and an internet connection. The pathologist can view the slide at any time just by logging into the system and can easily adjust it in three dimentional operating system.

\section{Conclusion}

Microscopes with camera are already used all around the world but this is very new technique in which the control of stage and movements of slides are controlled by pathologist. This is a novel idea that will help the remote areas to get specialist opinion regarding the critical cases in hematology, urine analysis, microbiology and histopathology on time.
It will reduce cost and time and pathologist can examine different cases from a places without moving anywhere so it will help a lot to patients and pathologist too. This equipment is totally dependent on the analysis of a pathologist, so if a diagnostic software is developed which helps them in detecting abnormal cells would be a great achievement and very helpful for them.

\section{Acknowledgement}

The author wishes to thank Mr. Asad Ali, CEO, Husaini Blood Bank for providing facility to carry out this research.

\section{References}

Arduino, 2014a. Arduino basics, available at http:// arduino. cc/en/ main/ software, last visited December 2014.

Arduino, 2014b. Arduino mega 2560 specifications, available at http://arduino.cc/en/Main/arduino BoardMega2560, last visited December 2014.

Eysenbach, G. 2001. What is e-health?. Journal of Medical Internet Research, 3: e20.

HTS, 2014. HyperTerminal Software trial package, available at http://www.hilgraeve.com/hyperterminal-triall/, last visited December 2014.

John, C.M., David, P.S., Joel, M.G.., Raquel, M.S., Kelly, J.B. 2009. Emergency department crowding, Part 2Barriers to reform and strategies to overcome them. Annals of Emergency Medicine, 53: 612-617.

Kamel Boulos, M.N., Wheeler, S. 2007. The emerging Web 2.0 social software: An enabling suite of sociable technologies in health and health care education. Health Information and Libraries Journal, 24: 2-23.

NILS, 2014. National Instruments Labview Software, available at http://www.ni.com/labview/, last visited December 2014.

NIVAS, 2014. National Instruments Vision Acquisition Softwere available at http://sine.ni.com/nips/cds/ view/p/lang/en/ nid/12892, last visited December 2014.

TVS, Team Viewer Software, available at http:// www. team viewer.com 